\title{
Report on the 4th International Workshop on Model-driven Robot Software Engineering (MORSE)
}

\author{
Sebastian Götz \\ Technische Universität Dresden, \\ Germany \\ sebastian.goetz@acm.org
}

\author{
Christian Piechnick \\ Technische Universität Dresden, \\ Germany \\ christian.piechnick@tu- \\ dresden.de
}

\author{
Andreas Wortmann \\ RWTH Aachen University, \\ Germany \\ wortmann@se-rwth.de
}

\begin{abstract}
The 4th edition of the international workshop on model-driven robot software engineering (MORSE) was held at the International Conference on Software Technologies: Applications and Foundations (STAF). The workshop took place in the city of Marburg, Germany, on the 21st of July 2017. The focus of this year's edition of the workshop was on scenario-based development and interaction modeling. In this report, we first present a synopsis of the workshop sessions before we highlight concerns raised in workshop's interactive discussion.
\end{abstract}

\section{INTRODUCTION}

With the advent of standard hardware platforms and software platforms for robots and the dynamics with which software ecosystems and app stores develop in application markets, the following research topics arise in the overlap of software engineering and robotics: (1) Model-driven engineering (MDE) for robotic systems, (2) software and app reuse for robotics, (3) end-user app development, (4) the compliance to legal and safety constraints, and (5) total cost of ownership. Model-driven engineering helps to design and develop complex systems by automating the development process concentrating on different levels of abstraction. With the advances in the robotic research communities and the increasing complexity of application scenarios for future robotic systems, model-driven techniques must be established to improve the quality (e.g., re-usability, reliability, maintainability) of the developed systems. Therefore, there is a need for a new paradigm of software and system development for robots. This suggests establishing a new joint community of researchers from robotics and software engineering.

The need and timeliness of this topic resulted in having a successful fourth edition of the MORSE workshop, which again took place as part of the International Conference on Software Technologies: Applications and Foundations (STAF) in Marburg, Germany. Previous editions were located at STAF 2014 (York, UK), STAF 2015 (L'Aquila, Italy), and RoboCup 2016 (Leipzig, Germany).

For this edition, we encouraged modeling-related submissions on robotic platforms, hardware and software abstractions, variability and reuse in robotics, computer vision and image processing, knowledge representation and reasoning, quality assurance, as well as validation and verification. Submissions could be either full papers or position papers. Full papers were expected to present original research, lessons learned from realizing a modeling approach, or experiences on transferring research prototypes into practice (15 pages). Position papers were expected to present well-argued vision or positions related to model-driven robotics software engineering (6 pages).
Overall, the workshop accepted six submissions addressing various concerns in robotics ranging from scenario-based formal specifications, formal behavior modeling techniques using UML, to (social) human-robot-interaction. Out of these, four submissions present original research and two sketch visions on model-driven robot software engineering. Despite the high number of concurrent workshops, it was attended by 12 participants.

\subsection{Organization Committee}

This edition of the MORSE workshop was organized by

- Sebastian Götz,

Technische Universität Dresden, Germany

- Christian Piechnick, Technische Universität Dresden, Germany

- Andreas Wortmann, RWTH Aachen University, Germany

\subsection{Program Committee}

We thank the members of the MORSE 2017 program committee, which are

- Colin Atkinson, University of Mannheim, Germany

- Herman Bruyninckx, KU Leuven, Belgium

- Kerstin Eder, University of Bristol, UK

- Frank J. Furrer, TU Dresden, Germany

- Kurt Geihs, Universitaet Kassel, Germany

- Diana Goehringer, Ruhr-University Bochum, Germany

- Nico Hochgeschwender, Bonn-Rhine-Sieg University of Applied Sciences, Germany

- Bernhard Jung, TU Freiberg, Germany

- Alexander Jungmann, IAV GmbH, Germany

- Jens Knoop, TU Vienna, Austria

- Lorenzo Natale, Instituto Italiano di Tecnologia, Italy

- Arne Nordmann, Robert Bosch GmbH, Germany

- Ralf Reussner, Karlsruhe Institute of Technology, Germany

- Bernhard Rumpe, RWTH Aachen University, Germany

- Davide Di Ruscio, Università degli Studi dell'Aquila, Italy

- Ulrik Schultz, University of Southern Denmark, Denmark

- Serge Stinckwich, IRD, France

- Cristina Vicente-Chicote, Universidad de Extremadura, Spain

- Heike Wehrheim, University of Paderborn, Germany

- Sebastian Wrede, CoR-Lab, Bielefeld University, Germany 


\section{THE WORKSHOP SESSIONS}

The MORSE 2017 workshop was organized into four sessions. After a keynote by Davide Di Ruscio on the role of models in engineering the software of robotic systems, two sessions of paper presentations, and an interactive discussion followed.

\subsection{Keynote: The Role of Models in Engineering the Software of Robotic Systems}

In his keynote, Davide Di Ruscio argued that despite robots being of our daily life for several decades (such as in manufacturing automation or in medical services), the software for robotic systems is typically developed in ad-hoc ways instead of following established engineering processes. This reduces reuse, configurability, and customizability of existing solutions. He continued by giving an overview on the state of the art and practice concerning solutions and open challenges in the engineering and management of robotics software. Model-driven engineering was presented as a promising technology to raise the level of abstraction, promote reuse, facilitate integration, boost automation, and promote early analysis in such a complex domain.

\subsection{Session 2: Scenario-based Development and Simulation}

The second session included three peer-reviewed articles.

The first presentation of this session, Synthesizing Executable PLC Code for Robots from Scenario-based GR(1) Specifications, Daniel Gritzner and Joel Greenyer presented a formal modeling method that supports software engineers the efficient development of robust robotic software through scenario-based requirements specifications. To this end, they present the scenario modeling language from whose models they synthesize code for programmable logic controllers (PLCs).

In the second presentation, Towards Synergistic Integration of Context-based and Scenario-based Development, Achiya Elyasaf, David Harel, Assaf Marron, and Gera Weiss also discussed leveraging scenario-based development to facilitate specification of robot behavior. To this end, they envision hierarchical scenario specifications based on life sequence charts that facilitate reusing specific scenario facets.

In the last presentation of this session, A Simulation Framework to Analyze Knowledge Exchange Strategies in Distributed Selfadaptive Systems, Christopher Werner, Sebastian Götz, and Uwe Aßmann presented a simulation platform for distributed and selforganizing multi-agent systems to explore different knowledge exchange strategies. The extensible platform facilitates specifying new types of constituent systems, communication mechanisms, and their interaction.

\subsection{Session 3: Behavior and Interaction Modeling}

The third session also included three peer-reviewed articles.

In their presentation, (An Example for) Formally Modeling Robot Behavior with UML and OCL, Martin Gogolla and Antonio Vallecillo discussed the lack of unified formal modeling notations and tools to address the many different aspects of robotics applications. They presented the application of UML with OCL to a chain of robotized arms that move parts in a production line and debate the possibilities that these high-level notations provide to describe the system's structure and behavior.
In the second presentation, Evaluating a Graph Query Language for Human-Robot Interaction Data in Smart Environments, Norman Köster, Sebastian Wrede, and Philipp Cimiano presented a collection of modeling languages for the efficient querying of longterm human-robot interaction data sets of embodied interaction in smart environments (EISE). To facilitate language usage, the authors present the EISE Query-Designer modeling workbench which is used in a comparative study on the benefits of the language collection of which they present preliminary results.

The last presentation of the session, Model-Driven Interaction Design for Social Robots, Gary Cornelius, Nico Hochgeschwender, and Holger Voos sketched a vision of a modeling language for human-robot interaction to address the challenges of engineering robot systems in the face of underspecified human behavior. Concerns of the envisioned modeling language were related to the scenario of deploying a museum guide robot which requires contributions from different stakeholders, including the museum employee responsible for deployment, the social scientists and human-robot interaction experts supporting the robots' interactions with museum visitors, and the software engineers.

\subsection{Session 4: Interactive Discussion}

The fourth session comprised an interactive discussion on the challenges robotics poses to software engineering and the opportunities model-driven engineering contributes to engineering robotics software. After collecting both, we clustered the opportunities and challenges. Based on these, the participants subsequently discussed interesting research directions. The discussion showed that challenges in model-driven robotics arise from its heterogeneity of platforms and tools, the lack of standardization, the inability to properly capture non-functional properties (such as safety or energy efficiency), and the complexities of modeling the systems' environments properly with respect to all relevant concerns (including dynamism and uncertainty).

The opportunities and chances in applying model-driven engineering to robotics emerge from the necessary increase of abstraction to ultimately support turn-key robotics solutions, enabling the formalization of solutions in a problem-specific granularity tailored towards the diverse experts participating in robotics projects, and the possibility to support provably-correct robotics software solutions based on adequate abstractions. Other great benefits in applying MDE to robotics stem from automation. These include continuous experimentation with models at runtime and generating vast configuration artifacts from more concise descriptions for specific applications. Modeling also lends itself to more comprehensive analyses through either simulation of formal verification. With proper automation mechanisms in place, the analyzed models also can serve as blueprint for software implementations.

From this, the participants derived the following interesting directions for future research:

- Developing techniques for self-adaptive models-at-runtime in the face of uncertainty.

- Addressing the cross-cutting concerns of safety and privacy through explicit and adjustable modeling techniques.

- Making the roles of model-driven engineering explicit to foster the separation of concerns in robotics projects.

- Investigating dependability in dynamic environments with adequate behavior modeling techniques and automated analyses. 


\section{DISCUSSION AND CONCLUSION}

Model-driven robot software engineering (MORSE) is a promising research field combining software engineering and robotics. Its objectives are to introduce model-driven engineering methodologies for the development of robot software. At the same time, formal methods should be transferred to robotics because "robot apps" need to be certified and verified. MORSE attempts to fill this gap.

This report shows the results of the 4th edition of our workshop. Two main conclusions can be drawn reflecting the program of the workshop and the active discussion of the participants. First, a wide range of approaches from model-driven software engineering shows up to be promising for improving the development of robotic applications (scenario-based development, model-based simulation, behavior modeling, etc.). Second, the MORSE research community is highly interdisciplinary and touches several other communities like, e.g.,, Models@run.time, safety engineering and model validation and verification to name but a few.

Thus, in retrospective to the lively 4th edition of the workshop, there is a clear need to continue with a fifth edition next year.

\section{Acknowledgments}

No workshop is successful by the efforts of only a few people. We would like to thank the members of the program committee who acted as anonymous reviewers and provided valuable feedback to the authors as well as the organizers of the STAF 2017 conference. 